\title{
SELEÇÃO GENÉTICA EM PROGÊNIES HÍBRIDAS DE COQUEIRO ${ }^{1}$
}

\author{
JOÃO TOMÉ FARIAS NETO ${ }^{2}$, PAULO MANOEL PONTES LINS ${ }^{3}$, \\ MARCOS DEON VILELADE RESENDE ${ }^{4}$, ANTÔNIO AGOSTINHO MULLER ${ }^{2}$
}

RESUMO - O presente trabalho objetivou realizar a seleção genética de indivíduos híbridos superiores de coqueiro a partir da avaliação de 1.080 plantas para produção de frutos e de albúmen fresco, visando a ao fornecimento de sementes e/ou clones melhorados para plantio no Pará. Empregaram-se o delineamento experimental de blocos ao acaso e os métodos Blupis e Blupis-Biefeitos, visando identificar em quais parcelas estão os melhores indivíduos e quantos indivíduos devem ser selecionados de cada parcela. Foram avaliados os caracteres número de frutos (NF), peso de albúmen fresco por fruto (PAFF) e peso de albúmen fresco por planta (PAFP), em nove safras anuais. As estimativas dos coeficientes de determinação genética entre médias de progênies variaram de $81,12 \%$ a 97,38\%, propiciando acurácias seletivas de 90,07\% a 98,68\% para a seleção entre famílias híbridas. A interação famílias x safras foi de grande magnitude para os caracteres NF e PAFP, conforme revelado pelas baixas $(40,77 \%$ a 63,36\%) correlações genotípicas através das safras. O procedimento Blupis - Biefeitos indicou a seleção de 66 plantas, sendo 48 da família PB113 e 18 da família PB111, propiciando intensidade de seleção de $6,1 \%$ e ganho genético de $9,95 \%$.

Termos para indexação: Cocos nucifera, BLUPIS, BLUP/REML, seleção, modelos mistos.

\section{GENETIC SELECTION IN HYBRID PROGENIES OF COCONUT PALM}

\begin{abstract}
The present study aimed the genetic selection of superior hybrid individuals of coconut palm from the evaluation of 1080 plants for the production of fruits and fresh albumen, aiming the supply of improved seeds and/or clones for plantation in Pará. The experimental design was randomized blocks and the methods Blupis and Blupis-Biefeitos were used to identify in which plots were the best individuals and how many individuals had to be selected in each plot. The characters evaluated were: number of fruits (NF), weight of fresh albumen per fruit (WFAF) and weight of fresh albumen for plant (WAFP), in nine annual harvests. The estimates of the coefficients of genetic determination between averages of progenies varied from $81.12 \%$ to $97.38 \%$, promoting a selective accuracy from $90.07 \%$ to $98.68 \%$ for the selection among hybrid families. The interaction families $\mathrm{x}$ harvests was of great magnitude for the characters NF and WFAF, as revealed by the low $(40.77 \% 63.36 \%)$ genotypic correlations throughout the harvests. The Blupis Biefeitos procedure indicated the selection of 66 plants, being 48 from the PB113 family and 18 from the PB111 family, propitiating a selection intensity of $6.1 \%$ and a genetic gain of $9.95 \%$.
\end{abstract}

Index terms: Cocos nucifera, BLUPIS, BLUP/REML, selection, mixed models.

\section{INTRODUÇÃO}

O coqueiro (Cocos nucifera) é uma espécie de grande importância econômica e social. Possui mais de 300 ecótipos e duas variedades principais: o coqueiro-gigante (variedade alógama) e o coqueiroanão (variedade autógama). Este último apresenta as subvariedades verde, vermelha e amarela (recessiva em relação à verde e à vermelha). A subvariedade vermelha apresenta dois tipos distintos fenotipicamente: o anão-da-malásia e o anão-dos-camarões (Siqueira et al., 1998).

Embora a clonagem para plantios comerciais em coqueiro não seja ainda uma tecnologia disponível, o emprego de sementes híbridas entre os tipos mencionados, para plantios em larga escala, é amplamente utilizado. Isto se deve à facilidade de obtenção dessas sementes por meio do uso das variedades anãs (autógamas) como genitores femininos e das técnicas de emasculação, conservação de pólen e polinização controlada. Devido à superioridade das cultivares híbridas, os

\footnotetext{
${ }^{1}$ (Trabalho 058-08). Recebido em: 14-03-2008. Aceito para publicação em: 13-01-2009.

${ }^{2}$ Pesquisadores da Embrapa Amazônia Oriental, Trav. Dr. Enéas Pinheiro, s/n Caixa Postal 48, 66095-100 Belém-PA., tome@cpatu.embrapa.br, amuller@cpatu.embrapa.br

${ }^{3}$ Socôco S.A. Agroindústria da Amazônia Rod. PA 252, km 38, Caixa Postal 15, CEP 68450-000, Moju-PA. Email:pmplins@uol.com.br

${ }^{4}$ Pesquisador da Embrapa Florestas, Estrada da Ribeira, km 11, Caixa Postal 319, 83411-000 Colombo-PR.
} 
programas de melhoramento do coco em vários países baseiam-se em um esquema de seleção recorrente recíproca (SRR) que visa a melhorar simultaneamente os híbridos Anão x Gigante e Gigante x Gigante, envolvendo dois ecótipos do coqueiro-gigante (Bourdeix et al.,1991 a e b; Baudouim et al., 1997). Em palmáceas, o esquema de SRR foi inicialmente desenvolvido para o melhoramento do dendê (Meunier \& Gascon, 1972; Cochard et al., 1993; Barcelos et al., 2000). Do ponto de vista do melhoramento genético do coqueiro, os caracteres mais importantes são o número de frutos, a quantidade de albúmen no fruto, a resistência a pragas e doenças, e a tolerância ao déficit hídrico (Siqueira et al., 1998).

No Estado do Pará, a cultura do coqueiro temse caracterizado pela baixa produtividade devido principalmente à implantação da cultura ocorrer com sementes de origem genética desconhecida, não existindo normalmente campos de matrizes de coqueiro, estabelecidos segundo os padrões técnicos para produção de sementes certificadas do próprio coqueiro-anão ou do coqueiro híbrido (Lins et al., 2003). Uma das estratégias adotadas pelos melhoristas, visando a aumentar a produtividade de coco, refere-se à avaliação e seleção de híbridos entre as variedades Anão x Gigante.

A avaliação genotípica compreende a estimação de componentes de variância (parâmetros genéticos) e a predição dos valores genotípicos. A experimentação de campo, via de regra, está associada ao desbalanceamento de dados devido a vários motivos, tais como perdas de plantas e parcelas, desiguais quantidades de sementes e mudas disponíveis por tratamento, rede experimental com diferentes números de repetições por experimento e diferentes delineamentos experimentais, nãoavaliação de todas as combinações genótipoambiente, dentre outros. Em função disso e da necessidade de inferências em nível genético e não fenotípico, o procedimento ótimo de avaliação genotípica refere-se ao REML/BLUP (máxima verossimilhança residual ou restrita/melhor predição linear não viciada). Estes procedimentos lidam naturalmente com o desbalanceamento conduzindo a estimações e predições mais precisas de parâmetros genéticos e valores genéticos, respectivamente. $\mathrm{O}$ procedimento BLUP maximiza a acurácia seletiva e, portanto, é superior ou, pelo menos, igual a qualquer outro método. As principais vantagens práticas do REML/BLUP são: permite comparar indivíduos ou variedades através do tempo (gerações e anos) e espaço (locais e blocos); permite a simultânea correção para os efeitos ambientais, estimação de componentes de variância e predição de valores genéticos; permite lidar com estruturas complexas de dados (medidas repetidas, diferentes anos, locais e delineamentos); pode ser aplicado a dados desbalanceados e a delineamentos não ortogonais; permite utilizar simultaneamente grande número de informações, provenientes de diferentes gerações, locais e idades, gerando estimativas e predições mais precisas (Henderson, 1984; Resende, 2002).

O procedimento BLUP tem sido usado no melhoramento de palmáceas para a seleção de progênies e indivíduos em pupunheira (Farias Neto $\&$ Resende, 2001 ) e palmeira-real (Bovi et al., 2003; 2004) e para a seleção de cruzamentos em dendê (Purba et al., 2001).

O método BLUP permite uma eficiente seleção de indivíduos, explorando todos os níveis de variabilidade genética e permitindo às melhores famílias e/ou aos melhores acessos contribuir com maior número de indivíduos. No entanto, em algumas espécies, este método não tem sido utilizado no melhoramento devido às dificuldades práticas em se obter os dados necessários de plantas individuais nos experimentos. Assim, há uma demanda por um procedimento prático que se assemelhe ao BLUP individual para aumento da eficiência do melhoramento dessas espécies. A estratégia de seleção, denominada BLUP individual simulado (BLUPIS), foi proposta por Resende e Barbosa (2006) para a seleção de indivíduos superiores em famílias de irmãos completos, sem a necessidade de avaliação individual de cada planta no experimento. Esse método permite a indicação do número de indivíduos a ser selecionado por família, o número total de clones para avanço com seleção e o número de famílias a contribuir com indivíduos selecionados, otimizando a seleção.

Com base no exposto, este trabalho objetiva realizar, por meio do método BLUPIS, a seleção genética de indivíduos híbridos superiores de coqueiro a partir da avaliação de 1.080 plantas, com base nas características produção de frutos e de albúmen fresco (sólido), visando ao fornecimento de clones e/ou sementes melhorados para plantio no Pará.

\section{MATERIAL E MÉTODOS}

O experimento foi instalado na Fazenda Socôco, localizada no Município de Moju-PA, situada a $02^{\circ} 07^{\prime} 00^{\prime \prime}$ de latitude sul e $48^{\circ}$ de longitude, distando $150 \mathrm{~km}$ da cidade de Belém. A altitude é de 30 metros, com pluviosidade média anual de 2.695 mm e temperatura média de $27^{\circ} \mathrm{C}$, umidade relativa 
do ar de $82 \%$ e constante iluminação solar. O solo da área experimental é um Latossolo Amarelo distrófico, textura argilo-arenosa e relevo suave. $\mathrm{O}$ plantio ocorreu no ano de 1984, e o início da avaliação ocorreu em 1989. O espaçamento adotado foi de 8,5 m x 8,5 m, em arranjo triangular equilátero. As adubações foram realizadas anualmente, de acordo com a diagnose foliar. Os tratos culturais, como capinas, foram realizados bimestralmente, e a colheita dos frutos ocorreu de 2 em 2 meses.

Os indivíduos fenotipados foram obtidos através de cruzamento de 6 progênies de irmãos germanos e avaliados em experimento em esquema de blocos casualizados. As progênies foram obtidas via polinização controlada, envolvendo materiais superiores. Os híbridos avaliados foram: PB 121 (Anão-amarelo-da-Malásia x Gigante-OesteAfricano); PB 111 (Anão-vermelho-de-Camarões x Gigante-Oeste-Africano); PB 141 (Anão-verde-doBrasil x Gigante-Oeste-Africano); PB 123 (Anãoamarelo-da-Malásia x Gigante-de-Renel); PB 132 (Anão-vermelho-da-Malásia x Gigante-da- Polinésia) e PB 113 (Anão-vermelho-de-Camarões x Gigantede-Renel), todos fornecidos pelo Institut de Recherches pour les Huiles et Oléagineux - IRHO, França.

Foram fenotipados 1.080 indivíduos, em 9 safras anuais, perfazendo um total de 9.720 observações analisadas. $\mathrm{O}$ experimento foi avaliado para os caracteres número de frutos (NF), peso de albúmen sólido por fruto (PAFF) e peso de albúmen sólido por planta (PAFP). Os dados foram analisados via metodologia de modelos lineares mistos (procedimento REML/BLUP).

Dessa forma, os parâmetros genéticos foram estimados via REML (máxima verossimilhança restrita), e os valores genotípicos ou médias genotípicas ajustadas de progênies, bem como os valores genéticos genotípicos de parcelas individuais foram estimados pelo procedimento BLUP (melhor predição linear não viciada), por meio do software Selegen-Reml/Blup (Resende, 2002). Foi utilizado o seguinte modelo estatístico para a avaliação genética:

$\mathrm{y}=\mathrm{Xb}+\mathrm{Zg}+\mathrm{Ts}+\mathrm{Qi}+\mathrm{Wp}+\mathrm{e}$, em que:

$\mathrm{y}, \mathrm{b}, \mathrm{g}, \mathrm{p}, \mathrm{s}, \mathrm{i}, \mathrm{e}=$ vetores de dados, de efeitos de blocos (fixos), de efeitos genotípicos de progênies de irmãos germanos (aleatório), de efeitos permanentes de parcelas (aleatório), de efeitos de safras (fixos), de efeitos de interação progênies x safra (aleatórios) e de erros aleatórios, respectivamente.

$\mathrm{X}, \mathrm{Z}, \mathrm{W}, \mathrm{T}, \mathrm{Q}=$ matrizes de incidência para $\mathrm{b}$, $\mathrm{g}, \mathrm{p}, \mathrm{s}$ e i, respectivamente.
Na derivação do BLUPIS, considerou-se que o valor genotípico verdadeiro dos indivíduos, tomando-se o indivíduo i do acesso j, é dado por: $\mu+g_{j}+g_{i j j}$, em que $\mu$ é a média geral, $g_{j}$ é o efeito genotípico do acesso $\mathrm{j}$ e $\mathrm{g}_{\mathrm{i} / \mathrm{j}}$ é o efeito genotípico do indivíduo $\mathrm{i}$ dentro do acesso $\mathrm{j}$.

O valor genotípico individual pode ser predito nas situações em que a observação fenotípica do indivíduo ij $\left(\mathrm{y}_{\mathrm{ij}}\right)$ é realizada. Neste caso, os BLUPs de dois indivíduos distintos ij e $\mathrm{kl}$, pertencentes aos acessos j e 1, podem ser comparados. Assim, o indivíduo i do acesso j será superior ao indivíduo $\mathrm{k}$ do acesso 1 se:

$\hat{\mu}+\hat{g}_{j}\left(1-h_{g d}^{2}\right)+h_{g d}^{2} y_{i j}>\hat{\mu}+\hat{g}_{l}\left(1-h_{g d}^{2}\right)+h_{g d}^{2} y_{k l}$

Percebe-se que as quantidades $\mathrm{h}_{\mathrm{gd}}^{2} \mathrm{y}_{\mathrm{ij}} \mathrm{e} \mathrm{h}_{\mathrm{gd}} \mathrm{y}_{\mathrm{kl}}$, ou seja, as frações de y ditadas pela herdabilidade dentro de acessos, independem dos valores genotípicos $\hat{g}_{j}$ e

$\hat{\mathrm{g}}_{1}$ dos acessos e são completamente aleatórias. Assim, $\mathrm{h}^{2}{ }_{\mathrm{gd}} \mathrm{y}_{\mathrm{ij}} \mathrm{e} \mathrm{h}^{2}{ }_{\mathrm{gd}} \mathrm{y}_{\mathrm{kl}}$ têm igual esperança matemática Desta forma, em média ou esperança matemática, o indivíduo do acesso j será superior se,

$$
\hat{\mu}+\hat{g}_{j}\left(1-h_{g d}^{2}\right)+h_{g d}^{2} y_{i j}>\hat{\mu}+\hat{g}_{l}\left(1-h_{g d}^{2}\right)+h_{g d}^{2} y
$$

ou seja, se $\hat{g}_{j} \quad \hat{g}_{1}\left(1-h_{g d}^{2}\right) /\left(1-h_{g d}^{2}\right)+h_{g d}^{2} y-h_{g d}^{2} y+\hat{\mu}-\hat{\mu}$ portanto, se $\hat{g}_{\mathrm{j}}>\hat{g}_{1}$, ou ainda se $\hat{g}_{\mathrm{j}}-\hat{\mathrm{g}}_{1}>0$ ou se $\hat{\mathrm{g}}_{\mathrm{j}} / \hat{\mathrm{g}}_{\mathrm{l}}>1$.

Desta forma, $\hat{\mathrm{g}}_{\mathrm{j}} / \hat{\mathrm{g}}_{1}$ indicou-se a taxa média de indivíduos superiores do acesso j em relação aos indivíduos do acesso l. Como exemplo, se $\hat{\mathrm{g}}_{\mathrm{j}} / \hat{\mathrm{g}}_{1}=$ 1,1 deverão ser selecionados 20 indivíduos do acesso le 22 indivíduos do acesso j para que o pior indivíduo do acesso j tenha o mesmo nível do pior indivíduo do acesso 1. Neste caso, esses 42 indivíduos deverão aproximar-se dos 42 melhores indivíduos que seriam selecionados pelo BLUP aplicado na seleção de indivíduos dos dois acessos.

Em resumo, a determinação do número de indivíduos a serem selecionados em cada acesso, usando a relação entre os efeitos genotípicos dos acessos simulou bem a seleção pelo procedimento BLUP individual. A expressão que determinou de forma dinâmica o número $\mathrm{n}_{\mathrm{j}}$ de indivíduos selecionados em cada acesso 1 foi dada por $\mathrm{n}_{1}=\left(\hat{\mathrm{g}}_{1} / \hat{\mathrm{g}}_{\mathrm{j}}\right) \mathrm{n}_{\mathrm{j}}$, em que $\hat{\mathrm{g}}_{\mathrm{j}}$ se refere ao efeito genotípico do melhor acesso e $n_{j}$ equivale ao número de indivíduos selecionados no melhor acesso. Todas as análises foram realizadas empregando-se o software Selegen-Reml/Blup (Resende, 2002; 2007).

\section{RESULTADOS E DISCUSSÃO}

Os resultados referentes às estimativas de 
parâmetros fenotípicos e genéticos para os três caracteres são apresentados na Tabela 1. As estimativas dos coeficientes de determinação genética em nível de parcelas individuais equivaleram a $15,86 \%, 41,14 \%$ e $30,44 \%$, para NF, PAFF e PAFP, respectivamente. Estimativas de herdabilidade em palmáceas são escassas. Soh et al. (2003) relatam herdabilidades variando de $22 \%$ a $36 \%$ para caracteres produtivos em dendê. Também Soh (1994), compilado por Resende (2002), relata os seguintes valores para a herdabilidade individual no sentido restrito: $35 \%, 20 \%$ e $15 \%$, para número de cachos, peso de cacho e produção de óleo, respectivamente. Estes valores são coerentes e próximos dos obtidos no presente trabalho. Ainda em dendê, vários autores (Corley et al., 1973; Hardon et al., 1972) têm relatado herdabilidades médias a altas $(21 \%$ a $65 \%)$ para caracteres de crescimento. Ramadasan et al. (1985) relataram alta herdabilidade para peso seco de raízes de coqueiro. Em açaizeiro, Farias Neto et al. (2007) relatam herdabilidades variando de $12 \%$ a $44,63 \%$. Todas essas estimativas denotam uma situação favorável para a seleção em palmáceas.

As estimativas dos coeficientes de determinação genética entre médias de progênies variaram de $81,12 \%$ a $97,38 \%$, propiciando acurácias seletivas de $90,07 \%$ a 98,68\% para a seleção entre famílias híbridas (Tabela 1). Estes valores são muito favoráveis à seleção (Resende \& Duarte, 2007). A interação famílias x safras foi de grande magnitude para os caracteres NF e PAFP, conforme revelado pelos altos coeficientes de determinação da interação famílias x safras e baixas $(40,77 \%$ a $63,36 \%)$ correlações genotípicas através das safras. Isto caracteriza uma situação de interação do tipo complexa (Vencovsky, 1987; Vencovsky \& Barriga, 1992), a qual é problemática para o melhorista. Nessa situação, a seleção de materiais mais estáveis através das safras é recomendada. Para o caráter PAFF, que apresentou maior determinação genética, a interação famílias x safras foi de baixa magnitude com correlação genotípica através das safras equivalendo a 99,19\%.

As estimativas de repetibilidade, obtidas simultaneamente aos coeficientes de determinação genética, apresentaram magnitudes de 42,35\%, $59,23 \%$ e $46,58 \%$ para NF, PAFF e PAFP, respectivamente (Tabela 1). Esses resultados revelam que, para uma avaliação confiável de NF e PAFP, são necessárias avaliações em um grande número de safras, da ordem de 5 ou mais (Farias Neto et al., 2003). Os valores de repetibilidade obtidos apresentam magnitudes médias conforme classificação de Resende (2002). Siqueira (1982) estimou o coeficiente de repetibilidade em $45,45 \%$ para produção de frutos em coqueiro.

Considerando que o caráter principal é PAFP, o qual congrega NF e PAFF, a seleção foi realizada para esse caráter, com base na produtividade simultânea em todas as safras. Os valores genotípicos preditos das melhores famílias para o caráter composto foram PB113 $(35,75)$, PB111 $(34,54)$ e PB141 (33,27). Essas apresentaram efeitos genotípicos positivos, ou seja, acima da média $(32,76)$. No entanto, na prática, deseja-se saber quais são os melhores indivíduos, dentre os 1.080 avaliados. Para isso, foi estudada detalhadamente cada parcela, por meio dos métodos Blupis e BlupisBiefeitos, visando a identificar em quais parcelas estão os melhores indivíduos e quantos indivíduos devem ser selecionados de cada parcela. Os resultados são apresentados na Tabela 2 .

O presente trabalho usa os procedimentos BLUPIS e BLUPIS - Biefeitos para o propósito da seleção de indivíduos, uma vez que dados de plantas individuais por parcela não estão disponíveis. $\mathrm{O}$ BLUPIS - biefeitos é um procedimento melhorado em relação àquele adotado por Soh \& Chow (1993) combinando as informações de progênies e de parcelas.

O procedimento BLUPIS recomendou a seleção de 84 indivíduos, sendo 48 da família PB113, 30 da família PB111 e 6 da família PB141. Por outro lado, o procedimento BLUPIS - Biefeitos indicou a seleção de 66 plantas, sendo 48 da família PB113 e 18 da família PB111 (Tabela 2). As intensidades de seleção equivaleram a 7,8\% e 6,1\% para o BLUPIS e BLUPIS - Biefeitos, respectivamente. O BLUPIS Biefeitos, além de conceitualmente mais adequado, conduziu a maior ganho genético predito, devido a propiciar uma seleção mais intensa. Assim, o mesmo será usado para inferências práticas. Tal método conduziu à seleção de indivíduos apenas nas duas melhores famílias. Já o BLUPIS selecionou indivíduos também na terceira família do ordenamento. Na Tabela 3, são apresentados os valores genotípicos preditos das melhores parcelas e ganho genético com a seleção pelo BLUPIS Biefeitos.

Para manutenção de uma adequada base genética e exploração adequada da variabilidade genética disponível, devem ser selecionados 66 indivíduos, sendo $8 \mathrm{em}$ cada uma das parcelas da família PB113 e 3 em cada uma das parcelas da família PB111. Essa seleção propiciará um ganho genético de 9,95\% no caráter PAFP. Esses 66 melhores indivíduos deverão ser usados como genitores para fornecimento de material genético melhorado ou serem submetidos a teste clonal para a identificação 
de clones superiores. Os indivíduos selecionados devem ser preferencialmente utilizados como clones. Podem também ser utilizados via sementes, porém o ganho genético será menor devido à segregação na progênie desses indivíduos híbridos.

TABELA 1 - Estimativas de parâmettros genéticos e fenotípicos para os caracteres número de frutos (NF), peso de albúmen fresco por fruto (PAFF) e peso de albúmen fresco por planta (PAFP), em famílias de irmãos completos de coqueiro avaliados no Estado do Pará.

\begin{tabular}{lccc}
\hline \multicolumn{1}{c}{ Parâmetro } & NF & PAFF (g) & PAFP (kg) \\
& & & 283,6102 \\
Variância genotípica entre progênies & 11,6304 & 121,7312 & 2,6258 \\
Variância permanente de parcelas & 19,4195 & 2,3144 & 2,6745 \\
Variância da interação progênies x safras & 16,8930 & 276,7276 & 5,4425 \\
Variância residual & 25,3685 & 684,3834 & 15,1945 \\
Variância fenotípíca & 73,3113 & 41,44 & 30,44 \\
Coeficiente de determinação de progênies nível parcela (\%) & 15,86 & 17,79 & 16,14 \\
Coeficiente de determinação de efeito de parcela (\%) & 26,49 & 0,0034 & 0,1760 \\
Coeficiente de determinação de efeito interação progênie x safras & 0,2304 & 97,38 & 91,25 \\
Coeficiente de determinação de progênies nível médias (\%) & 81,12 & 98,68 & 95,53 \\
Acurácia na seleção de progênies (\%) & 90,07 & 99,19 & 63,36 \\
Correlação genotípica através das safras (\%) & 40,77 & 59,23 & 46,58 \\
Repetibilidade de parcelas individuais (\%) & 42,35 & 8,0459 \\
Coeficiente de variação residual & 8,0420 & 83756 \\
\hline Média geral & 83,2175 & 395,5978 & 32,7633 \\
\hline
\end{tabular}

TABELA 2 - Ordem das melhores parcelas e número de indivíduos selecionados pelos procedimentos Blupis e Blupis biefeitos para o caráter peso de albúmen fresco por planta, em famílias de irmãos completos de coqueiro avaliadas no Estado do Pará.

\begin{tabular}{ccc}
\hline Híbrido Parcela & $\begin{array}{c}\text { Número de } \\
\text { indivíduos } \\
\text { selecionados por } \\
\text { parcela pelo } \\
\text { Blupis }\end{array}$ & $\begin{array}{c}\text { Número de indivíduos } \\
\text { selecionaods por } \\
\text { parcela pelo Blupis- }\end{array}$ \\
& Biefeitos \\
\end{tabular}

\begin{tabular}{lccl}
\hline 113 & 34 & 8 & 8 \\
113 & 2 & 8 & 8 \\
113 & 19 & 8 & 8 \\
113 & 10 & 8 & 8 \\
113 & 25 & 8 & 8 \\
113 & 17 & 8 & 8 \\
111 & 28 & 5 & 3 \\
111 & 32 & 5 & 3 \\
111 & 22 & 5 & 3 \\
111 & 1 & 5 & 3 \\
111 & 16 & 5 & 3 \\
111 & 9 & 5 & 3 \\
141 & 14 & 1 & 0 \\
141 & 21 & 1 & 0 \\
141 & 31 & 1 & 0 \\
141 & 11 & 1 & 0 \\
141 & 4 & 1 & 0 \\
141 & 27 & 1 & 0 \\
\hline Total & & 84 & 66 \\
\hline & & &
\end{tabular}

TABELA 3 -Valores genotípicos preditos das melhores parcelase ganho genético com a seleção pelo Blupis - biefeitos para o caráterpeso de albúmen fresco por planta, em famílias de irmãos completos de coqueiro avaliadas no Estado do Pará.

\begin{tabular}{ccccc}
\hline Híbrido & Parcela & $\begin{array}{c}\text { Valor } \\
\text { genotípico } \\
\text { predito }\end{array}$ & $\begin{array}{c}\text { Número de } \\
\text { indivíduos } \\
\text { selecionados } \\
\text { por parcela } \\
\text { pelo Blupis- } \\
\text { Biefeitos }\end{array}$ & $\begin{array}{c}\text { Ganho } \\
\text { genético } \\
\text { em cada } \\
\text { parcela } \\
(\%)\end{array}$ \\
\hline 113 & 34 & 35,88 & 8 & 13,87 \\
113 & 2 & 35,83 & 8 & 13,83 \\
113 & 19 & 35,76 & 8 & 13,78 \\
113 & 10 & 35,75 & 8 & 13,77 \\
113 & 25 & 35,75 & 8 & 13,77 \\
113 & 17 & 35,63 & 8 & 13,68 \\
111 & 28 & 34,69 & 3 & 4,95 \\
111 & 32 & 34,61 & 3 & 4,71 \\
111 & 22 & 34,57 & 3 & 4,60 \\
111 & 1 & 34,51 & 3 & 4,41 \\
111 & 16 & 34,49 & 3 & 4,35 \\
111 & 9 & 34,45 & 3 & 4,23 \\
\hline Valor médio do ganho genético & & 9,95 \\
\hline
\end{tabular}




\section{CONCLUSÕES}

1-Os procedimentos BLUPIS e BLUPISbiefeitos selecionaram diferentes números de famílias e indivíduos.

2-Ganho genético considerável pode ser obtido com a seleção dos 66 indivíduos envolvendo duas famílias selecionadas pelo método BLUPISbiefeitos para peso de albúmen fresco por planta.

3-Foram detectadas interações famílias $\mathrm{x}$ safras do tipo complexa, para número de frutos $\mathrm{e}$ peso de albúmen fresco por planta, conforme revelado pelas baixas correlações genotípicas através das safras.

\section{REFERÊNCIAS}

BARCELOS, E.; NUNES, C. D. M.; CUNHA, R. N. V. Melhoramento genético e produção de sementes comerciais de dendezeiro. In: VIEGA, I. J. M.; MULLER, A. A. A cultura do dendezeiro naAmazônia Brasileira. Belém: Embrapa, 2000. p. 145-174.

BAUDOUIM,L.; BARIL, C.; CLEMENTDEMANGE, A.; LEROY, T.; PAULIN, D. Recurrent selection of tropical tree crops. Euphytica, Dordrecht, v. 96, n. 1, p. 101-114, 1997.

BOURDEIX, R.; MEUNIER, J.; N'CHO, Y. P. Une stratégie de sélection du cocotier Cocus nucifera. II. Amélioration des hybrides Grand x Grand. Oléagineux, Paris, v.46, n. 7, p. 267-282, 1991a.

BOURDEIX, R; MEUNIER, J.; N'CHO, Y. P. Une stratégie de sélection du cocotier Cocus nucifera. II. Amélioration des hybrides Nain x Grand. Oléagineux, Paris, v.46, n. 10, p. 361-374, 1991 b.

BOVI, M. L. A.; RESENDE, M. D. V.; SPIERING, S. H. Genetic parameters estimation in King palm through a mixed mating system model. Horticultura Brasileira, Brasília, v. 21, n. 1, p. 93-98, 2003.

BOVI, M. L. A.; RESENDE, M. D. V. ; SÁES, L. A. ; UZZO, R. P. Genetic analysis for sooty mold resistance and heart of palm yield in Archontophoenix. Scientia Agricola, Piracicaba, v. 61, n. 2, p. 178-184, 2004.

COCHARD, B.; NOIRET, J. M.; BAUDOUIN, L.; AMBLARD, P. Second cycle de sélection récurrente réciproque chez le palmier à huile Elais guineensis.
Résultat des tests d'hybrides Deli x La Mé. Oléagineux, Paris, v. 48, n. 11, p. 441-451, 1993.

CORLEY, R.H.V.; HARDON, J.J.; OOI, S.C. Some evidence for genetically controlled variation in photosynthetic rate of oil palm seedlings. Euphytica, Dordrecht, v. 22, n. 1, p. 48-55, 1973.

FARIAS NETO, J. T.; LINS, P. M. P.; MULLER, A. A. Estimativas dos coeficientes de repetibilidade para produção de fruto e albúmen fresco em coqueiro híbrido. Pesquisa Agropecuária Brasileira, Brasília, v. 38, n. 10, p. 1237-1241, 2003.

FARIAS NETO, J. T.; RESENDE, M. D. V. Aplicação da metodologia de modelos mistos (REML/BLUP) na estimação de componentes de variância e predição de valores genéticos em pupunheira (Bactris gasipaes). Revista Brasileira de Fruticultura, Jaboticabal, v. 23, n. 2, p. 320-324, 2001.

FARIAS NETO, J. T.; RESENDE, M. D. V. de; OLIVEIRA, M. S. P.; SANTOS, N. S. A.; NOGUEIRA, O. L.; MULLER, A. A. Avaliação genética de progênies de polinização aberta de açaí (Euterpe oleracea) e estimativas de parâmetros genéticos. Cerne, Lavras, v.13, p. 376-383, 2007.

HARDON, J.J.; OOI, S.C.; CORLEY, R.H.V. Analysis of growth in oil palm.2. Estimation of genetic variances of growth parameters and yield of fruit bunches. Euphytica, Dordrecht, v.21, n. 2, p. 252264, 1972.

HENDERSON, C. R. Aplications of linear models in animal breeding. Guelph: University of Guelph, 1984. $462 \mathrm{p}$.

LINS, P. M. P.; FARIAS NETO, J. T.; MULLER, A. A. Avaliação de híbridos de coqueiro (Cocus nucifera L.) para produção de frutos e de albúmen fresco. Revista Brasileira de Fruticultura, Jaboticabal, v. 25, n. 3, p. 468-470, 2003.

MEUNIER, J.; GASCON, J. P. Le schema général d'amelioration du palmier à huile à IRHO. Oléagineux, Paris, v. 27, p. 1-12, 1972.

PURBA, A.R.; FLORI, A.; BAUDOUIN, L.; HAMON, $\mathrm{S}$. Prediction of oil palm agronomic performances using the best linear unbiased predictor (BLUP). Theoretical and Applied Genetics, Berlin, v. 102, p.787-792, 2001. 
RAMADASAN, A.; KASTURI-BAI， K.V.; SHIVASHANKAR, S.; VIJAYAKUMAR, K. Heritability of seedling vigour in coconut palm. Journal of Plantation Crops, Kerala, v.13, n.2, p. 136138, 1985.

RESENDE, M. D. V. de. Genética biométrica e estatística no melhoramento de plantas perenes. Brasília: Embrapa Informação Tecnológica, 2002. 975 p.

RESENDE, M. D. V. de. Matemática e estatística na análise de experimentos e no melhoramento genético. Colombo: Embrapa Florestas, 2007. 561 p.

RESENDE, M. D. V. de; BARBOSA, M.H.P. Selection via simulated individual BLUP based on family genotypic effects in sugarcane. Pesquisa Agropecuária Brasileira, Brasília, v.41, n. 3, p. 421429, 2006.

RESENDE, M. D. V. de; DUARTE, J. B. Precisão e controle de qualidade em experimentos de avaliação de cultivares. Pesquisa Agropecuária Tropical, Goiânia, v. 37, n.3, p. 182-194, 2007.

SIQUEIRA, E. R. Coeficiente de repetibilidade da produção de frutos de coqueiro comum. Pesquisa Agropecuária Brasileira, Brasília, v. 17, n. 3, p. 573 574, 1982.
SIQUEIRA, E. R.; RIBEIRO, F. E.; ARAGÃO, W. M.; TUPINAMBÁ, E. A. Melhoramento genético do coqueiro. In: FERREIRA, J.M.S.; WARWICK, D.R.N.; SIQUEIRA, L.A. A cultura do coqueiro no Brasil. Brasília: Embrapa-SPI, 1998. p. 73-98.

SOH, A. C. Ranking parents by best linear unbiased prediction (BLUP) of breeding values in oil paml. Euphytica, Dordrecht, v. 76, n. 1-2, p. 13-21, 1994.

SOH, A.C.; CHOW, C.S. Index selection utilizing plot and family information in oil palm. Elaeis, v.5, p.2737, 1993

SOH, A.C. ; GAN, H.H.; WONG, G.; HOR, T.Y.; TAN C.C. Estimates of within family genetic variability for clonal selection in oil palm. Euphytica, Dordrecht, v.133, p.147-163, 2003.

VENCOVSKY, R. Herança quantitativa. In: Paterniani, E.; Viegas, G.P. (Ed.). Melhoramento e produção de milho. 2.ed. Campinas: Fundação Cargill, 1987.

VENCOVSKY, R.; BARRIGA, P. Genética biométrica no fitomelhoramento. Ribeirão Preto: Sociedade Brasileira de Genética, 1992. 486 p. 